\title{
2D Guide Wire Tracking during Endovascular Interventions
}

\author{
S.A.M. Baert and W.J. Niessen \\ Image Sciences Institute, University Medical Center Utrecht \\ Rm E 01.334, P.O.Box 85500, 3508 GA Utrecht, The Netherlands \\ \{shirley, wiro\}@isi.uu.nl
}

\begin{abstract}
A method to extract and track the position of a guide wire during endovascular interventions under X-ray fluoroscopy is presented and evaluated. The method can be used to improve guide wire visualization in the low quality fluoroscopic images and to estimate the position of the guide wire in world coordinates. A two-step procedure is utilized to track the guide wire in subsequent frames. First a rough estimate of the displacement is obtained using a template matching procedure. Subsequently, the position of the guide wire is determined by fitting a spline to a feature image in which line-like structures are enhanced. In the optimization step, the influence of the scale at which the feature is calculated is investigated. Also, the feature image is calculated both on the original image and on a preprocessed image in which coherent structures are enhanced. Finally, the influence of explicit endpoint detection is studied. The method is evaluated on 267 frames from 10 sequences. Using the automatic method, the guide wire could be tracked in $96 \%$ of the frames, with a greater accuracy than three observers. Endpoint detection improved the accuracy of the tip assessment, which was better than $1.3 \mathrm{~mm}$.
\end{abstract}

\section{Introduction}

Endovascular interventions are rapidly advancing as an alternative for invasive classical vascular surgery. During these interventions a guide wire is inserted into the groin and is advanced under fluoroscopic guidance. Accurate positioning of the guide wire with respect to the vasculature is a prerequisite for a successful procedure. Especially during neuro-interventions positioning the guide wire correctly is difficult because of the complexity of the vasculature and narrowness of the blood vessels, causing an increase of intervention time and radiation exposure.

In this paper an automated method for guide wire tracking during endovascular interventions is considered. The method can be used to improve visualization of the guide wire, potentially enabling a reduction in radiation exposure. It can also be used to detect the position of the guide wire in world coordinates which enables registration with preoperatively acquired images, so as to provide a navigation tool for radiologists. 
There is relatively little literature on tracking guide wires from $2 \mathrm{D}$ fluoroscopy images. The possibility to use guide wire tracking to extract information regarding myocardial function is evaluated in [6]. However, tracking was only performed in a single frame and not in time. Other research has been directed towards active tracking of guide wires and catheters to control their position inside the human body using external devices 911 , or to reconstruct $3 \mathrm{D}$ catheter paths [2. There has been a considerable amount of work on the enhancement and extraction of curved line structures. In medical imaging, it is used to extract anatomical features such as (centerlines of) blood vessels, e.g. 45,710.

In this paper, a multiscale method to extract and track guide wires using a spline minimization approach in a feature image is presented. The influence of the scale at which the feature is determined, the use of coherence enhancing diffusion as preprocessing step and specific endpoint detection are studied. The proposed method has been validated by comparing the results to tracings obtained by three observers.

\section{Methods}

In order to represent the guide wire, a spline parameterization is used. For all experiments in this paper, we used a third order B-spline curve.

To determine the position of the spline in frame $n+1$ if the position in frame $n$ is known, a two-step procedure is introduced. First, a rigid translation is determined to capture the rough displacement of the spline. Next, a spline optimization procedure is performed in which the spline is allowed to deform for accurate localization of the guide wire. These steps can be understood as a coarse-to-fine strategy, where the first step ensures a sufficiently good initialization for the spline optimization.

\subsection{Rigid Transformation}

In order to obtain a first rough estimate of the displacement, a binary template is constructed based on the position in the present frame. The best location of this template in the new frame is obtained by determining the highest cross correlation of the frame with a certain search region in this image (or features derived from it (section 2.3) ). Only rigid translations are considered in this step.

\subsection{Spline Optimization}

After performing the rigid translation, the spline is optimized under internal and external forces. The internal constraints are related to the geometry of the curve and influence the length (first derivative of the B-spline) and the bendedness (second derivative of the spline). The parameters for the curvature are set sufficiently large to avoid strange shapes of the spline and sufficiently small to ensure that the internal forces only have a small influence on the total spline energy. For the external forces, the image intensity or a feature image derived 
from it (see section 2.3) is used. The spline contains four or five control points and one hundred sample points. The spline is optimized using Powell's direction set method [8]. In order to have a minimum length of the spline, the energy $E$ is defined cumulatively for lengths smaller than $L$, and relatively for larger lengths:

$$
E=\int_{0}^{l} \frac{E(s)}{\max (l, L)} d s
$$

Here $l$ is the length of the spline. In all experiments in this paper a minimum length of $L=60$ pixels is used.

\subsection{External Image Force}

Using original images for the matching and optimization steps, the guide wire can not effectively be tracked due to presence of other objects in the image and/or due to the low signal-to-noise ratio of the images. Therefore a filter which enhances line-like structures of the correct orientation is considered. Also, the use of coherence enhancing diffusion, as a preprocessing step in order to reduce noise while maintaining line-like structures, is evaluated.

Coherence-Enhancing Diffusion. To reduce the noise in the fluoroscopic images a nonlinear diffusion technique is used, in which coherent flow-like textures are enhanced. The diffusion equation is given by

$$
\partial_{t} I(\mathbf{x} ; t)=\nabla \cdot(D \nabla I(\mathbf{x} ; t))
$$

where $D$ denotes a diffusion tensor that can be chosen such that coherent structures are enhanced [12].

This diffusion tensor depends on the structure tensor $\mathbf{M}$, given by

$$
\mathbf{M}=\nabla I(\mathbf{x} ; \tau) \nabla I(\mathbf{x} ; \tau)^{T},
$$

with eigenvalues $\mu_{1}$ and $\mu_{2}\left(\mu_{1} \geq \mu_{2}\right)$ and the corresponding orthonormal eigenvectors $\mathbf{v}_{\mathbf{1}}$ and $\mathbf{v}_{\mathbf{2}}$. The gradient is computed at scale $\sigma_{n}=\sqrt{2 \tau}, \tau>0$. Using diffusion based on the structure tensor not only the amount but also the direction of diffusion can be regulated. Smoothing along the coherence direction $\mathbf{v}_{\mathbf{2}}$ with a diffusity $\lambda_{2}$ which increases with respect to the coherence $\left(\mu_{1}-\mu_{2}\right)^{2}$, gives an enhancement of the coherent structures in an image. This is achieved by constructing $\mathbf{D}$ from the following system of orthonormal eigenvectors

$$
\begin{gathered}
\mathbf{v}_{\mathbf{1}} \| \nabla I(\mathbf{x} ; \tau), \\
\mathbf{v}_{\mathbf{2}} \perp \nabla I(\mathbf{x} ; \tau),
\end{gathered}
$$

and eigenvalues

$$
\begin{aligned}
& \lambda_{1}=\alpha, \\
& \lambda_{2}= \begin{cases}\alpha & \text { if } \mu_{1}=\mu_{2}, \\
\alpha+(1-\alpha) \exp \left(\frac{-C}{\left(\mu_{1}-\mu_{2}\right)^{2}}\right) & \text { else }\end{cases}
\end{aligned}
$$


with $C \geq 0$ and $\alpha \in(0,1)$ which keeps $\mathbf{D}$ uniformly positive definite. Figure 1 shows an example of a frame preprocessed using coherence enhancing diffusion.
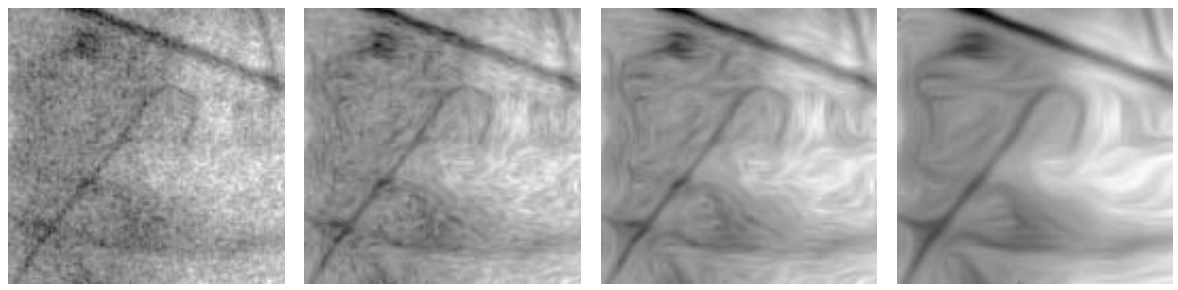

Fig. 1. From left to right: The original image and image preprocessed using coherence enhancing diffusion with $t=5, t=20$ and $t=100$.

Feature Image. To determine the optimal spline position, a feature image is derived in which line-like structures are enhanced. The feature image is determined on the original image and the image preprocessed with coherence enhancing diffusion. Hereto, the eigenvalues $\lambda_{1}, \lambda_{2}$ of the Hessian matrix calculated at scale $\sigma$ are considered:

$$
\lambda_{1,2}(\mathbf{x}, \sigma)=\frac{1}{2}\left(I_{x x}+I_{y y} \pm \sqrt{\left(I_{x x}-I_{y y}\right)^{2}+4 I_{x y}^{2}}\right)
$$

where $I_{x y}$ represents the convolution with the scaled Gaussian derivative. On line-like structures the largest absolute eigenvalue $\lambda_{1}$ has a large output. Since we are interested in dark elongated structures on a brighter background, only positive values of $\lambda_{1}$ are considered; pixels with negative values of $\lambda_{1}$ are set to zero. The feature image is subsequently constructed by inverting this image since the optimization is based on a minimum cost approach.

To effectively attract the guide wire only to line structures with similar orientation, we also use directional information in the optimization scheme. Hereto, the inner product between the spline and the orientation of the feature is used given by

$$
O\left(\hat{x}_{i}\right)=\lambda_{1}\left(\hat{\mathbf{e}}_{2} \cdot \hat{x}_{i}\right)
$$

where $\hat{\mathbf{e}}_{2}$ is the normalized eigenvector corresponding to $\lambda_{2}$ and $\hat{x}_{i}$ is the normalized first derivative of the spline in sample point $i$.

To be sensitive to guide wires of different width, and to reduce sensitivity to noise, the feature image can be calculated at multiple scales $(\sigma)$. This can also be used to enable a coarse-to-fine optimization strategy.

\subsection{Explicit Endpoint Detection}

After the fitting procedure, the endpoint of the spline is not necessarily positioned on the endpoint of the guide wire. In order to determine the endpoint, 
the length of the guide wire is increased at the tip by setting $L$ equal to $l+\Delta L$, see Equation 1 while fixing the tail position. This procedure is carried out iteratively such that the endpoint of the spline is advanced beyond the endpoint of the guide wire. From the final spline position, a graph is constructed presenting the likeliness $P(i)$ of each sample point $i$ on the spline to represent the guide wire endpoint. Two criteria are used to determine this likeliness, viz. the proximity to the previous endpoint position, and the derivative of the feature image in $i$ along the spline:

$$
P(i)=\exp \left(-\frac{\left\|\Delta x_{n(i), n-1}\right\|-\left\|\Delta x_{n-1, n-2}\right\|}{\sigma_{\text {prox }}{ }^{2}}\right) \cdot \nabla F(i)\left(\sigma_{\text {grad }}\right)
$$

where the first term compares the displacement $\Delta x_{n(i), n-1}$ of a candidate endpoint $i$ in the current frame with the displacement $\Delta x_{n-2, n-1}$ in the previous frame, favoring similar displacements using a Gaussian weigthing function with standard deviation $\sigma_{\text {prox }}$. The value of $\sigma_{\text {prox }}$ has been obtained from analysis of the changes in displacements that were observed in a large number of image sequences. $\nabla F$ represents the gradient of the feature image. In order to be robust to noise, a coarse-to-fine approach is used. First at a large scale $\sigma_{\text {grad }}$ the gradient maximum is determined whereas precise localization is achieved at smaller scales.

\section{Evaluation}

The method was applied on ten image sequences, with a sequence length between 14 and 50 frames, with a total of 267 frames. The image series were acquired on a H5000, H3000 and a BV5000 X-ray fluoroscopy system (Philips Medical Systems, Best, the Netherlands). Only J-tipped guide wires were used during the interventions.

To evaluate the automatic method, a golden standard is constructed for every dataset. Therefore, three observers have manually outlined the guide wire in every image. This process was repeated after two weeks to limit the dependence between tracings. These six manually obtained paths are averaged to determine the average observer path $\bar{C}$ ("golden standard"), to which all individual paths are compared. Intra-observer variability can then be measured and inter-observer variability is defined as the distance betweeen individual tracings of observers and the golden standard. Likewise, to determine the accuracy of the automated method the distance between the determined spline and the golden standard is measured. A method to construct the average path $\bar{C}$ and a definition of the distance between two paths is required, for which more details can be found in [1].

\section{Results}

The performance of the method is evaluated on feature images calculated on both the original images and images preprocessed with coherence enhancing diffusion. 
Also the influence of explicit endpoint detection is investigated. An example of the tracking results is shown in Figure 2, All parameters in the experiments were kept fixed for all image sequences. Coherence enhancing diffusion was applied with $C=1, \alpha=0.001$, and the evolution was stopped at $t=20$. The feature
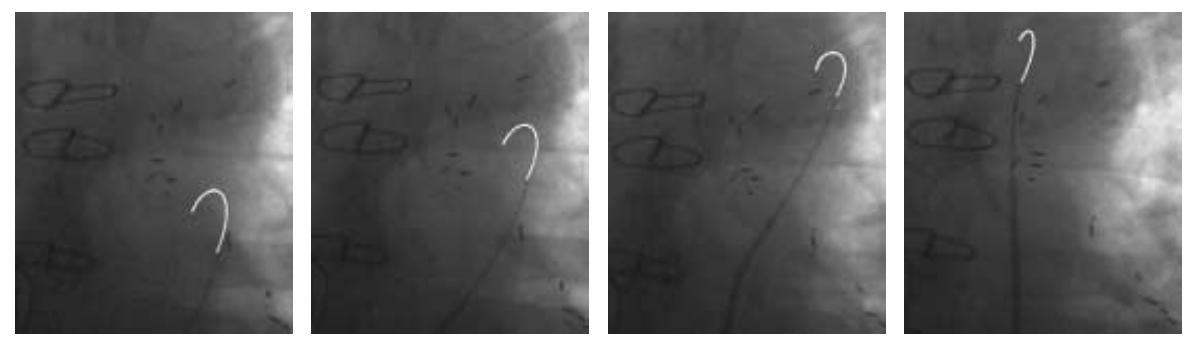

Fig. 2. An image sequence of the thorax with in white the parametrized spline representing the guide wire. The method was applied on the feature image in which the eigenvalues of the Hessian matrix were calculated with $\sigma=1.5$.

enhancement step is carried out on three different scales $(\sigma=1,1.5$ and 3 pixel units) which implies that a total of twelve possibilities are investigated. For the endpoint detection two scales are used ( $\sigma_{\text {grad }}=3$ and 1.5 pixels) and $\sigma_{\text {prox }}$ is set to 7 pixels. Table 1 shows the intra and inter-observer variability and the results of the method without coherence enhancing diffusion as preprocessing step.

The mean distance is the average distance between the corresponding parts of the splines as described in the previous section. The tip distance is the distance between the endpoint of the golden standard and the endpoint of the automatic determined spline. Between the brackets, the maximum distance is represented. It can be observed from Table 1 that best results are obtained at a scale $\sigma=1.5$ pixel units. With these settings, the mean error of the automatic method (0.92 pixels) is smaller than the inter-observer variability (1.04 pixels). Moreover, the method only requires initialization in one frame, and reproducibility was better than intra-observer variability.

Owing to motion blur, the guide wire can sometimes become invisible in a number of sequences, which causes the spline to be incorrectly placed. At a scale $\sigma=1.5$ pixel units the number of outliers in our evaluation was ten frames (out of 267 frames) occurring in four sequences (out of ten sequences). These failures appeared mostly in a single frame. Without manual intervention, the guide wire was tracked correctly in the subsequent frames. For the tip distance we can observe that the intra- and inter-observer variability (1.09 and 1.46 pixels, respectively) is smaller than the error by the automatic tracking method ( 4.38 pixels) for $\sigma=1.5$. This error improved to 3.07 pixels by applying explicit endpoint detection, however the mean distance increased slightly in this case. Since the pixelsize is approximately $0.4 \mathrm{~mm}$, the tip error is smaller than 1.3 millimeters. 
Table 1. The mean intra- and inter-observer variability and the mean result of the automatic method using the Hessian feature filter with and without specific endpoint detection.

\begin{tabular}{lllll}
\hline \hline & $\begin{array}{c}\text { Mean distance } \\
\text { [pixels] }\end{array}$ & $\begin{array}{l}\text { Tip distance } \\
{[\text { pixels }]}\end{array}$ & $\begin{array}{l}\text { Mean distance } \\
\text { with Endpoint }\end{array}$ & $\begin{array}{l}\text { Tip distance } \\
\text { with Endpoint }\end{array}$ \\
\hline \hline Intra observer & $0.66[1.46]$ & $1.09[1.77]$ & & \\
\hline Inter observer & $1.04[2.19]$ & $1.46[2.44]$ & & \\
\hline$\sigma=1.0$ & $1.26[3.10]$ & $5.81[9.35]$ & $1.22[2.15]$ & $4.20[8.56]$ \\
\hline$\sigma=1.5$ & $0.92[1.49]$ & $4.38[8.20]$ & $1.13[2.02]$ & $3.07[4.20]$ \\
\hline$\sigma=3.0$ & $1.05[1.47]$ & $5.70[10.92]$ & $1.29[2.15]$ & $3.80[5.77]$ \\
\hline \hline
\end{tabular}

Table 2 shows the results obtained using the feature image calculated with the coherence enhancing diffusion method as a preprocessing step prior to enhanceing line-like structures. We can observe that the distance between the automatic determined spline and the golden standard is smaller than the inter-observer variability for all three different scales. The number of total outliers for this method was 10 frames out of 267 frames for $\sigma=1.5$. The failures appeared mostly in a single frame, for example due to motion blur in the image sequence. Results did not degrade significantly when altering the scale in the step of enhancing the guide wire. Specific endpoint detection improved the error for the tip distance, but it slightly increased the mean distance.

Table 2. The mean result of the automatic method using the coherence enhancing diffusion filter with and without specific endpoint detection.

\begin{tabular}{lllll}
\hline \hline & $\begin{array}{c}\text { Mean distance } \\
\text { [pixels] }\end{array}$ & $\begin{array}{c}\text { Tip distance } \\
{[\text { pixels }]}\end{array}$ & $\begin{array}{l}\text { Mean distance } \\
\text { with Endpoint }\end{array}$ & $\begin{array}{l}\text { Tip distance } \\
\text { with Endpoint }\end{array}$ \\
\hline \hline$\sigma=1.0$ & $1.04[1.74]$ & $4.95[12.50]$ & $1.31[2.32]$ & $3.60[5.88]$ \\
\hline$\sigma=1.5$ & $0.96[1.67]$ & $4.33[6.18]$ & $1.13[2.16]$ & $3.26[5.26]$ \\
\hline$\sigma=3.0$ & $0.92[1.50]$ & $5.33[11.89]$ & $1.13[2.15]$ & $4.01[7.48]$ \\
\hline \hline
\end{tabular}

\section{Discussion}

A method has been developed to track the guide wire automatically in fluoroscopic guided interventions. During these interventions, 12.5 frames per second are acquired, so manual outlining is not an option. The method is based on a spline optimization in an image where line-like structures with correct orientation are enhanced, with or without coherence enhancing diffusion as a preprocessing 
step and with or without explicit endpoint detection. In order to assess whether the proposed method is sufficiently accurate, tracings of observers were acquired in 267 frames. Both with and without preprocessing, the accuracy of spline localization was better than inter-observer variability and the method detected the guide wire correctly in $96 \%$ of the frames. Outliers occurred mainly in one single frame owing to motion blur. Given the high temporal resolution (12.5 frames/second) missing one frame does not hamper the interventional radiologist. In fact, in most of these frames the guide wire is not visible to the radiologist either. The tip of the guide wire could be localized within an accuracy of approximately $1.3 \mathrm{~mm}$, using explicit endpoint localization. Whereas the use of coherence enhancing diffusion did not significantly improve the accuracy if the proper scale was used for enhancing line-like structures, the results were more robust with respect to changing the scale parameter. This indicates that the use of coherence enhancing diffusion is useful for robustness in clinical practice.

\section{References}

1. C.M. van Bemmel, W.J. Niessen, O. Wink, B. Verdonck and M.A. Viergever, Blood Pool Agent CE-MRA: Improved Arterial Visualization of the Aortoiliac Vasculature in the Steady-State Using First-Pass Data, in Proceedings of MICCAI 2001, Lecture Notes in Computer Science Vol. 2208, pp. 699-706.

2. H.-J. Bender, R. Männer, C. Poliwoda, S. Roth, M. Walz, Reconstruction of 3D Catheter Paths from 2D X-ray Projections, in Proceedings of MICCAI 1999, Lecture Notes in Computer Science Vol.1679, pp 981-989.

3. M. Kass, A. Witkin, D. Terzopoulos, Snakes: Active contour models, International Journal of Computer Vision 1987, Vol. 1, No. 4, pp. 321-331.

4. A.K. Klein, F. Lee, and A.A. Amini Quantitative Coronary Angiography with Deformable Spline Models, IEEE Trans. on Med. Imaging 1997, Vol.16, 5: pp. 468-482.

5. R. Kutka and S. Stier, Extraction of Line Properties Based on Direction Fields, IEEE Transactions on Medical Imaging 1996, Vol.15, No.1: pp. 51-58.

6. D. Palti-Wasserman, A.M. Brukstein, R.P. Beyar, Identifying and Tracking a Guide Wire in the Coronary Arteries During Angioplasty from X-Ray Images, IEEE Transactions on Biomedical Engineering 1997, Vol.44, No.2: pp. 152-164.

7. R. Poli, G. Valli, An Algorithm for Real-time Vessel Enhancement and Detection, Computer Methods and Programs in Biomedicine 1997 No. 52: pp. 1-22.

8. W.H. Press, S.A. Teukolsky, W.T. Vetterling, B.P. Flannery, Numerical Recipes in $C$ : The art of scientific computing, 1992.

9. J. Ragasa, N. Shan, R.W. Watson, Where antecubital catheters go: a study under fluoroscopic control, Anesthesiology 1989, No. 71: pp. 378-380.

10. Y. Sato, S. Nakajima, H. Atsumi, S. Yoshida, Th. Koller, G. Gerig, R. Kikinis, Three-dimensional Multi-scale Line Filter for Segmentation and Visualization of Curvilinear Structures in Medical Images, Med. Im. An. 1998, Vol.2, 2: pp. 143-168.

11. H. Starkhammar, M. Bengtsson, D.A. Kay, Cath-Finder Catheter Tracking System: a new device for positioning of central venous catheters. Early experiments from implantation of brachial portal systems, Acta Anaes.Scand.1990,No.34: pp.296-300.

12. J. Weickert, Coherence-Enhancing Diffusion Filtering, International Journal of Computer Vision 1999, Vol.31, No.2/3, pp 111-127. 